\title{
Prognostic Factors for Recurrence in Esophageal Cancer Patients Treated With Neoadjuvant Therapy and Surgery: A Single-institution Analysis
}

Misbah Khan ${ }^{1}$, Namra Urooj ${ }^{2}$, Aamir Ali Syed ${ }^{2}$, Shahid Khattak ${ }^{2}$, Ather Kazmi ${ }^{3}$, Mohammad I. Ashraf ${ }^{2}$, Sadaf Batool ${ }^{1}$

1. Surgery, Shaukat Khanum Memorial Cancer Hospital and Research Centre, Lahore, PAK 2. Surgical Oncology, Shaukat Khanum Memorial Cancer Hospital and Research Centre, Lahore, PAK 3. Radation Oncology, King Faisal Specialist Hospital and Research Centre, Riyadh, SAU

Corresponding author: Namra Urooj, namra_urooj@yahoo.com

\section{Abstract}

\section{Background}

The purpose of this study is to analyze potential predisposing factors for a higher risk of recurrence in our esophageal cancer patients managed with neoadjuvant chemotherapy, radiation therapy, and surgery, and to determine their impact on disease-free survival (DFS) and time to recurrence.

\section{Methods}

A total of 154 of 232 patients staged T1b to T4a managed electively at our institute from July 2005 through July 2015 with a tri-modality approach were retrospectively evaluated. Basic demographic, clinical, radiological, operative, and pathological disease-related parameters, along with waiting time for surgery and type of neoadjuvant modality used, were assessed as potential risk factors. The primary endpoint was the impact of these on the risk of recurrence. The secondary endpoint was to study their relation on DFS and time to recurrence.

\section{Results}

The recurrence rate in this particular study was $33.1 \%$ over a median follow-up of 35 months (interquartile range $=19-50.3$ ). The median time to recurrence was 12 months, and $94 \%$ of recurrences occurred within two years. The median DFS was 33 months, and the one- and three-year DFS was $90 \%$ and $72 \%$, respectively. On univariate and multivariate analysis, a complete pathological response (hazard ratio [HR]: 3.8, 95\% confidence interval [CI]: 1.41-10.11), negative resection margins (HR: 5.9, 95\% CI: 1.69-20.45), and a low nodal index (HR: 6.3, 95\% CI: 1.37-28.67 for an index of 0.1-0.2; and HR: 15.2 , 95\% CI: 0.96-241.73 for an index of $>0.2)$ were found as statistically significant $(\mathrm{P}=<0.05)$ for risk to recurrence. In addition to these

Received 04/09/2020 Review began 05/01/2020 Review ended 05/05/2020 Published 05/14/2020

\section{() Copyright 2020}

Khan et al. This is an open access article distributed under the terms of the Creative Commons Attribution License CC-BY 4.0., which permits unrestricted use, distribution, and reproduction in any medium, provided the original author and source are credited. three, a low comorbidity index ( $\mathrm{P}=0.03$; HR: 3.5; 95\% CI: 1.16-10.52) was an individual positive predictor of DFS.

\section{Conclusions}

A complete pathological response, low nodal index, and margin-negative resection were the identified predictors of freedom from recurrence, with a better DFS and a low comorbidity index as additional indicators of prolonged DFS.

Categories: Radiation Oncology, Oncology

Keywords: risk factors, recurrence, neoplasm recurrence, disease-free survival, esophageal cancer, prognosis, recurrence local, recurrence locoregional

\section{Introduction}

Esophageal cancer is a complex disease associated with a very high recurrence rate. It is the sixth most common cause of cancer-related death worldwide [1]. Combined modality treatment with neoadjuvant chemotherapy and radiation therapy (XRt) followed by surgical resection is the current standard of care associated with a better survival and disease control as compared with either treatment modality alone [2,3]. The reported overall five-year survival after perioperative chemotherapy is $38 \%$, with a disease-free survival (DFS) of $34 \%$ at five years [4]. On the contrary, a three-year overall survival rate of $50 \%$ after esophagectomy along with neoadjuvant chemoradiation therapy and a locoregional control rate reaching up to $80 \%$ at three years have been reported [5-8]. Nevertheless, the reported recurrence rate after the radical tri-modality treatment strategy is still up to $35 \%$ to $42 \%[3,9]$. 
Our hospital is a high-volume center for esophageal cancer management. As presented in our adult patient population, esophageal cancer is the sixth most common overall and the fifth most common cancer among solid organ malignancies seen at our center [10]. Standard management protocol for resectable esophageal cancer T1b and above is neoadjuvant chemotherapy and XRt followed by surgical resection and en bloc lymph node dissection. However, it is not infrequent to see recurrent disease in these patients after treatment completion. The data from our center have shown a recurrence rate of $39 \%$ in this treated set of the population [11]. In the absence of any measurable tools or pre-established defining factors to predict the occurrence of treatment failure, it is imperative to study the patient populations who develop a poor outcome [12]. A study in this population could pave the way in segregating patient groups at a higher risk of recurrence, allowing the development of enhanced surveillance programs for such identified clusters with a final goal of defining strategies at its prevention.

The goal of this study was to analyze potential predisposing factors for a higher risk of recurrence in our esophageal cancer patients managed with neoadjuvant chemotherapy, XRt, and surgery, and to determine their impact on DFS and time to recurrence.

\section{Materials And Methods}

An overview of this study was initially presented as an abstract at the 16th Shaukat Khanum Cancer Symposium, 2017, and at the National Comprehensive Cancer Network Annual Conference, 2019 (unpublished abstract: Misbah Khan, Prognostic Factors for Recurrence in Esophageal Cancer Patients Treated with Neoadjuvant Therapy and Surgery, NCCN 2019 Annual Conference, Improving the Quality, Effectiveness and Efficiency of Cancer Care, March 22, 2019) [13]. The full details of the study are presented herein. Our study was a retrospective analytic design. All potentially resectable esophageal cancer patients at our institute from July 2005 through July 2015 with an elective multidisciplinary management plan of neoadjuvant treatment followed by esophageal resection were evaluated. After a formal exemption status for the study was granted by the Institutional Review Board, basic demographics, clinical, radiological, and pathological disease-related parameters were assessed through the hospital information system for a total of 232 patients.

Patients with an emergency esophagectomy $(n=8)$ and hence no neoadjuvant treatment, histopathology indicating something other than adenocarcinoma or squamous cell cancer, and a Siewert type 3 tumor location on initial endoscopy were excluded. Moreover, patients with metastatic disease at presentation and before completion of treatment were considered ineligible. For documentation of recurrence, patients with a less than one year of follow-up period, including those with early mortality due to causes other than a recurrent disease, were excluded. The last date of follow-up for all the patients was June 8, 2018. Of 187 patients, 15 died earlier than six months, and 18 were lost to follow-up (unable to complete a minimum oneyear follow-up) and were omitted from the final data.

The variables explored for a relationship with recurrence in addition to the basic patient-related demographic variables were tumor histological type, pathological grade, location, initial radiological TNM (tumor, node, metastasis) stage, pathological nodal index, completeness of margin status, type of neoadjuvant modality used, type of surgical procedure performed, post-operative course (major class III/IV complications and extended hospital stay), and duration between the completion of neoadjuvant treatment and surgical procedure performed (Table 1).

\begin{tabular}{|c|c|c|c|}
\hline \multirow[t]{2}{*}{ Variable } & Total & Recurrence & No Recurrence \\
\hline & N (\%), 154 (100) & $\mathrm{N}(\%), 51(33.1)$ & $\mathrm{N}(\%), 103$ (66.9) \\
\hline \multicolumn{4}{|l|}{ Gender } \\
\hline Men & 79 (51.3) & $28(54.9)$ & $51(49.5)$ \\
\hline Women & 75 (48.7) & $23(45.1)$ & $52(50.5)$ \\
\hline \multicolumn{4}{|l|}{ Comorbidity index } \\
\hline $0-1$ & 139 (90.3) & $43(84.3)$ & $96(93.2)$ \\
\hline$>1$ & $15(9.7)$ & $8(15.7)$ & $7(6.8)$ \\
\hline \multicolumn{4}{|l|}{ Histological subtype } \\
\hline Adenocarcinoma & $35(22.7)$ & $15(29.4)$ & 20 (19.4) \\
\hline Squamous cell carcinoma & 119 (77.3) & 36 (70.6) & $83(80.6)$ \\
\hline \multicolumn{4}{|l|}{ Pathological tumor grade } \\
\hline Well-differentiated & $17(11)$ & $3(5.9)$ & $14(13.6)$ \\
\hline
\end{tabular}




\section{Cureus}

\begin{tabular}{|c|c|c|c|}
\hline Moderately & $100(64.9)$ & $36(70.6)$ & $64(62.1)$ \\
\hline Poorly & $34(22.1)$ & $12(23.5)$ & $22(21.4)$ \\
\hline \multicolumn{4}{|l|}{ Tumor initial radiological stage } \\
\hline Early (stage I-Ilb) & $36(23.4)$ & $9(17.6)$ & $27(26.2)$ \\
\hline Late (stage Illa and above) & $118(76.6)$ & $42(82.4)$ & 76 (73.8) \\
\hline \multicolumn{4}{|l|}{ Tumor location } \\
\hline Mid esophageal & $104(67.5)$ & $36(70.6)$ & $68(66.0)$ \\
\hline Distal/GOJ Siewert type 1 & $40(26)$ & $8(15.7)$ & $32(31.1)$ \\
\hline GOJ Siewert type 2 & $10(6.5)$ & $7(13.7)$ & $3(2.9)$ \\
\hline \multicolumn{4}{|l|}{ Neoadjuvant type } \\
\hline Carboplatin-paclitaxel based XRt & $53(34.4)$ & $16(31.4)$ & $37(35.9)$ \\
\hline 5-FU based XRt & $81(52.6)$ & $23(45.1)$ & $58(56.3)$ \\
\hline Magic protocol & $15(9.7)$ & 10 (19.6) & $5(4.9)$ \\
\hline Incomplete & $5(3.2)$ & $2(3.9)$ & $3(2.9)$ \\
\hline \multicolumn{4}{|l|}{ Pathological response to neoadjuvant } \\
\hline CR & $85(55.2)$ & $17(33.3)$ & $68(66.0)$ \\
\hline Without CR & $69(44.8)$ & $34(66.7)$ & $35(34.0)$ \\
\hline \multicolumn{4}{|l|}{ Type of surgery } \\
\hline Conventional & $63(40.9)$ & $25(49.0)$ & $38(36.9)$ \\
\hline Minimally invasive & 65 (42.2) & $21(41.2)$ & $44(42.7)$ \\
\hline Hybrid & 26 (16.9) & $5(9.8)$ & $21(20.4)$ \\
\hline \multicolumn{4}{|l|}{ Oncological resection margin } \\
\hline Negative & $122(79.2)$ & $29(56.9)$ & $93(90.3)$ \\
\hline Positive & $32(20.8)$ & $22(43.1)$ & $10(9.7)$ \\
\hline \multicolumn{4}{|c|}{ Post-operative/major complications class III/IVII } \\
\hline No & $130(8.4)$ & $42(82.4)$ & $88(85.4)$ \\
\hline Yes & 24 (15.6) & $9(17.6)$ & $15(14.6)$ \\
\hline \multicolumn{4}{|l|}{ Hospital stay } \\
\hline$<11$ days & $118(76.6)$ & 39 (76.5) & 79 (76.7) \\
\hline$>11$ days & $36(23.4)$ & $12(23.5)$ & $24(23.3)$ \\
\hline \multicolumn{4}{|l|}{ Nodal index } \\
\hline$<0.1$ & $129(83.8)$ & $32(62.7)$ & $97(94.2)$ \\
\hline $0.1-0.2$ & $15(9.7)$ & $11(21.6)$ & $4(3.9)$ \\
\hline$>0.2$ & $10(6.5)$ & $8(15.7)$ & $2(1.9)$ \\
\hline \multicolumn{4}{|c|}{ Interval between neoadjuvant and surgery } \\
\hline Standard & $57(37.0)$ & 18 (35.2) & 39 (37.9) \\
\hline Delayed & $94(61.0)$ & $31(60.8)$ & $63(61.2)$ \\
\hline \multicolumn{4}{|l|}{ Mean + standard error of the mean } \\
\hline Age(years) & $52.6+0.86$ & $54.7+1.46$ & $51.6+1.06$ \\
\hline
\end{tabular}




\section{Cureus}

\begin{tabular}{|llll|}
$<55$ years & $83(53.9)$ & $25(49.0)$ & $58(56.3)$ \\
$>55$ years & $71(46.1)$ & $26(51.0)$ & $45(43.7)$ \\
BMI $\left(\mathrm{kg} / \mathrm{m}^{2}\right)$ & $22.4+0.35$ & $21.9+0.58$ & $22.5+0.43$ \\
$18-25$ & $98(63.6)$ & $29(56.8)$ & $69(67)$ \\
$>25$ & $36(23.4)$ & $11(21.6)$ & $25(24.3)$ \\
$<18$ & $20(13)$ & $11(21.6)$ & $9(8.7)$ \\
Mean number of lymph nodes harvested & $13.5+0.44$ & $12.2+0.72$ & $14.1+0.54$ \\
Length of follow-up (in months) & $36.8+1.63$ & $19.8+1.53$ & $45.5+1.81$ \\
\hline
\end{tabular}

\section{TABLE 1: Patient demographic and tumor characteristics}

Co-morbidity classification as per the Charlson Comorbidity Index.

IIClavien-Dindo Classification system for post-operative complications.

FU, fluorouracil; CR, complete pathological response; GOJ, gastroesophageal junction; XRt, radiation therapy; body mass index

For an optimal oncological and surgical quality assessment purpose, we have included variables of margin status, a pathological response to neoadjuvant treatment, and nodal index instead of pathological $\mathrm{T}$ and $\mathrm{N}$ stage. Table 2 shows the detailed pre- and post-neoadjuvant stage distribution and the extent of downstaging achieved in our study group.

\begin{tabular}{|c|c|c|c|}
\hline & Total & Recurrence & No Recurrence \\
\hline & N (\%), 154 (100) & $\mathrm{N}(\%), 51$ (33.1) & $\mathrm{N}(\%), 103$ (66.9) \\
\hline \multicolumn{4}{|c|}{ Tumor initial T stage } \\
\hline T1a, T2 & $10(6.5)$ & $2(3.9)$ & $8(7.8)$ \\
\hline T3 & $107(69.5)$ & $33(64.7)$ & 74 (71.8) \\
\hline T4b & $37(24)$ & $16(31.4)$ & $21(20.4)$ \\
\hline \multicolumn{4}{|c|}{ Tumor initial $\mathrm{N}$ stage } \\
\hline NO & $37(24)$ & $12(23.5)$ & $25(24.3)$ \\
\hline N1, N2 & $117(76)$ & $39(76.5)$ & $78(75.7)$ \\
\hline \multicolumn{4}{|c|}{ Pathological T stage } \\
\hline T0 & 85 (55.2) & 17 (33.3) & $68(66.0)$ \\
\hline T1-3 & $67(43.5)$ & 32 (62.8) & $35(34.0)$ \\
\hline T4 & $2(1.3)$ & $2(3.9)$ & $0(0.0)$ \\
\hline \multicolumn{4}{|c|}{ Pathological N stage } \\
\hline No & $125(81.2)$ & $30(58.8)$ & 95 (92.2) \\
\hline N1 & $18(11.7)$ & $12(23.5)$ & $6(5.8)$ \\
\hline N2 & $8(5.2)$ & $6(11.8)$ & $2(1.9)$ \\
\hline N3 & $3(1.9)$ & $3(5.9)$ & $0(0.0)$ \\
\hline
\end{tabular}

\section{TABLE 2: Detailed stage distribution pre and post-neoadjuvant treatment}


radiological stage was divided into early stages 1 and 2 and late-stage 3A and above [14]. A circumferential margin of less than $1 \mathrm{~mm}$ and a longitudinal margin of $<1 \mathrm{~cm}$ on the final histopathology report was defined as positive or close. Waiting time of more than 6 weeks after neoadjuvant chemotherapy and more than 12 weeks after chemoradiation was considered as delayed.

The primary endpoint was to evaluate the impact of these prognostic factors on the risk of recurrence in the group, which developed recurrence, as compared with the patient group who remained recurrence-free. The secondary endpoint is to study the relation of the aforementioned factors on DFS and time to recurrence.

For all middle to lower esophageal cancers, staged $\mathrm{T} 1 \mathrm{~b}$ and above, standard management protocol at our center is neoadjuvant induction chemotherapy (5-fluorouracil [FU]/platinum-based or newer carboplatinpaclitaxel based regimen) followed by concurrent chemoradiation therapy (45 Gy in 25 fractions), with an assessment and proceed to surgery at six to eight weeks. Some cases of adenocarcinoma of the true cardia (Siewert type 2) on final histopathological assessment also received perioperative chemotherapy on MAGIC protocol as per multidisciplinary board decision based on their initial diagnostic assessment (endoscopic ultrasound and contrast-enhanced computed tomography [CT]). Additionally, a positron emission tomography (PET)-CT in all patients and a staging laparoscopy for Siewert type 2 adenocarcinoma was performed to rule out metastatic elements before the start of a curative intent treatment protocol.

Patients were monitored through follow-up at regular intervals after completion of treatment with both surgery and oncology clinics. The standard guideline for follow-up was the first visit at two weeks after surgery to review and discuss the histopathology report followed by visits at one and three months. Then patients were monitored through follow-up at six-month intervals for the initial two years post-surgery and yearly thereafter for the next three years. A CT of the chest and abdomen with contrast was repeated at a one-year interval unless prompted for by symptoms or signs of recurrence. Endoscopy was only performed for the evaluation of any significant dysphagia associated symptoms. If recurrence was suspected by the above investigations, additional confirmatory tests including PET-CT and biopsy are advised.

\section{Statistical analysis}

It was a retrospective analytic study design. All data were analyzed using SPSS Statistics for Windows, Version 20.0. (IBM Corp., Armonk, NY, USA). Pearson's chi-square test for categorical variables and one-way analysis of variance for continuous variables, along with binary logistic regression analysis, were used for establishing univariate and multivariate associations of all prognostic factors for the assessment of risk to recurrence. Cox regression analysis was used to assess time to recurrence and DFS and the impact of prognostic factors on these two variables. For regression analysis, continuous variables were dichotomized according to their mean values or split into sub-groups depending on their clinical significance. For all practical purposes, a P-value of 0.05 or less was considered significant.

\section{Results}

A total of 154 patients with a median follow-up of 35 months (interquartile range [IQR $]=19-50.3$ months) were further evaluated. The median age of our selected patient population was 53.5 years (IQR $=47-60$ years), and their body mass index (BMI) was $21.7(\mathrm{IQR}=19.6-25.1)$. The median length of hospital stay was nine days $(\mathrm{IQR}=8-11$ days), waiting time for surgery from the date of completion of neoadjuvant treatment was 13 weeks (IQR = 11-18 weeks), and we had a median nodal harvest of 13 (IQR = 10-17).

The length of follow-up was considerably longer for the group without recurrence (median: 43.5 months; $\mathrm{IQR}=32-59.3)$ as compared with the patients who develop a recurrence (median: 16 months; $\mathrm{IQR}=12-25.8$ ).

The recurrence rate in this particular study is $33.1 \%$. Depending on patients' initial mode of presentation, $60.8 \%$ were distant metastatic recurrences (31 cases), including peritoneal disease and non-regional lymph nodes. While $21.6 \%$ (11 cases) were found to have a locoregional disease relapse, the remaining $17.6 \%$ (nine cases) had both locoregional and metastatic recurrent disease.

On univariate and multivariate linear logistic regression analysis after controlling for all included variables in the study, a complete pathological response (CR), negative resection margins, and a low nodal index were found as statistically significant $(P \leqslant 0.05)$ predictors of freedom from recurrence (Table 3). Overall DFS on Cox regression survival plots showed similar trends (Figure 1), with a median DFS of 33 months and a oneand three-year DFS of $90 \%$ and $72 \%$, respectively.

Potential Prognostic Factors

Univariate Analysis

Odds

Ratio
$\mathrm{P}$

value

alysis

\section{Multivariate Analysis}

Adjusted Odds

Ratio
Pvalue
$95 \% \mathrm{Cl}$

Age $<55$ years vs 


\section{Cureus}

$>55$ years

Gender: men vs.

Women

BMI: 18-25 vs.

$>25$

$<18$

Comorbidity index: $0-1$ vs.

$>1$

Histological subtype: SCCA vs.

Adenocarcinoma

Tumor grade: well differentiated vs.

Moderately differentiated

2.63

Poorly differentiated

Tumor initial radiological stage: Early vs.

Late (IIla and above)

Tumor location: mid-esophageal vs.

Siewert type 1

Siewert type 2

Neoadjuvant type: carboplatin-paclitaxol based XRt

vs.

5-FU based XRt

Magic protocol

Incomplete

Type of surgery: conventional vs.

Minimally invasive

Hybrid

Waiting time for surgery: standard vs.

Delayed

Length of hospital stay: $<11$ days vs.

$>11$ days

Post-operative: no vs.

Major complications

Complete pathological response vs.

Partial or no

Oncological complete resection vs.

Margin-positive

7.06

.34

2.64

2.55

1.82

5

1.66

0.92

4.63

1.54

0.73

0.36

1.07

1.01

1.29

3.89
0.393

$0.68-2.63 \quad 1.33$

0.584

$0.48-3.65$

$0.529 \quad 0.41-1.58 \quad 0.66$

0.005

$1.69-20.45$

$\begin{array}{llll}1.05 & 0.914 & 0.46-2.40 & 1.17\end{array}$

0.804

$0.33-4.18$

$0.057 \quad 0.97-7.18 \quad 2.38$

0.257

$0.53-10.6$

$0.08 \quad 0.87-7.49 \quad 4.13$

0.108

$0.73-23.24$

$0.13 \quad 0.83-3.98 \quad 0.65$

0.636

$0.11-3.93$

$0.149 \quad 0.71-9.75 \quad 1.08$

0.931

$0.21-5.54$

0.862

$0.14-5.29$

$0.61-$
10.65

0.85

$(2.862$

0.078

$0.88-10.62$

$0.237 \quad 0.71-3.85 \quad 3.06$

0.078

0.03

$0.04-0.84$

$\begin{array}{llll}0.47 & 0.092 & 0.19-1.13 & 0.17\end{array}$

0.904

$0.04-17.74$

18.08

0.83

$0.823 \quad 0.43-1.96 \quad 0.85$

$0.762 \quad 0.29-2.50$

$0.56 \quad 0.20-19.34$

$0.014 \quad 1.36-$

1.97

(1)

$0.73 \quad 0.02-13.64$

0.652

$0.24-$

10.13

0.57

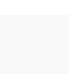

0.75

$0.22-2.94$

$0.385 \quad 0.35-1.49 \quad 0.81$

$0.204 \quad 0.07-1.77$

$\begin{array}{lll}0.07 & 0.12-1.09 & 0.35\end{array}$

0.164

$0.73-6.17$

$0.859 \quad 0.53-2.16 \quad 2.13$

$0.738 \quad 0.36-4.18$

$0.975 \quad 0.46-2.24 \quad 1.23$

$0.74 \quad 0.32-5.07$

$\begin{array}{lll}0.583 & 0.52-3.19 & 1.27\end{array}$

0

$1.91-7.91 \quad 3.78$

$0.008 \quad 1.41-10.11$

Nodal index $<0.1$ vs. 


\section{Cureus}

$0.1-0.2$

$>0.2$
8.34

12.13
0.001

0.002
2.4828.01

2.4560.07
6.26

0.018

TABLE 3: Univariate and multivariate regression analysis for risk of recurrence

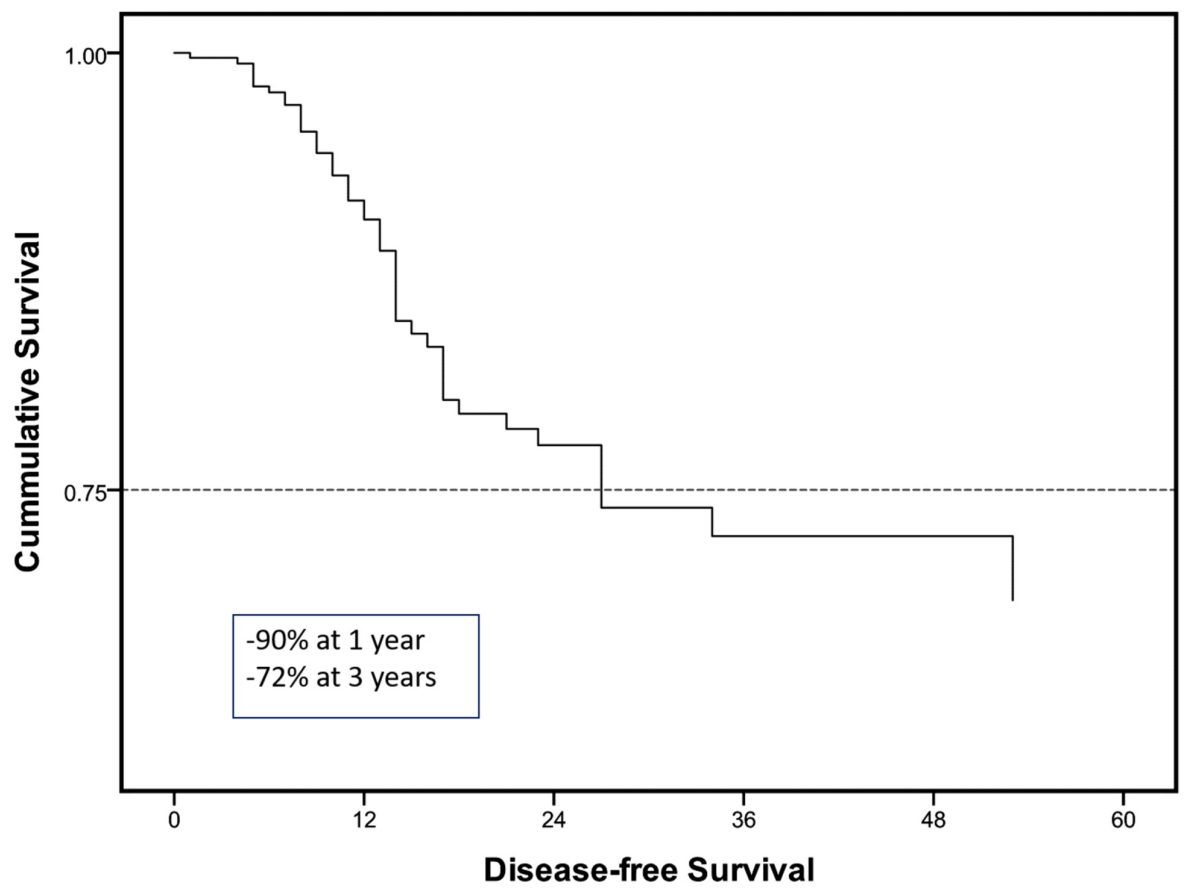

FIGURE 1: Cox regression analysis for overall disease-free survival at the mean of covariates

In addition to the three positive prognostic factors for a risk to recurrence, a low comorbidity index was also an individual predictor of DFS (Figures 2-4). The median time to recurrence was 12 months (Table 4). Most of the recurrences occurred within the first two years (94\%) and 99\% within 27 months after surgery

(Figure 5). Only a couple of cases were reported late at 34 and 53 months; one was adenocarcinoma and the other squamous, managed with magic protocol and 5-FU based therapy, respectively. However, on the Cox regression model, none of the individual factors had any statistically significant impact on time to recurrence (Table 5). 


\section{Cureus}

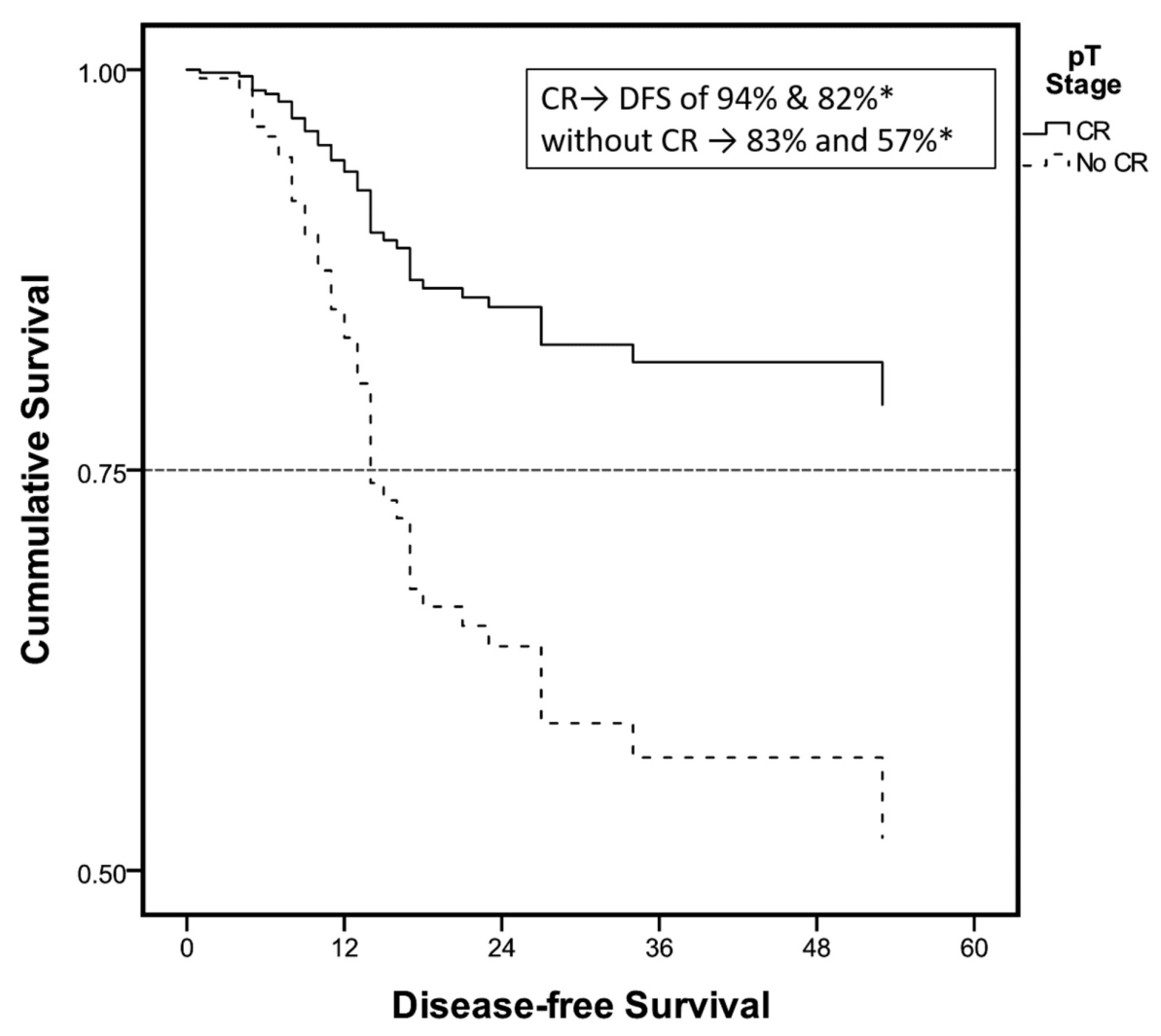

FIGURE 2: Cox regression survival curves: subgroup analysis

Survival curve: pathological stage.

${ }^{*} \mathrm{DFS}$ at one and three years.

CR, Cox regression; DFS, disease-free survival. 


\section{Cureus}

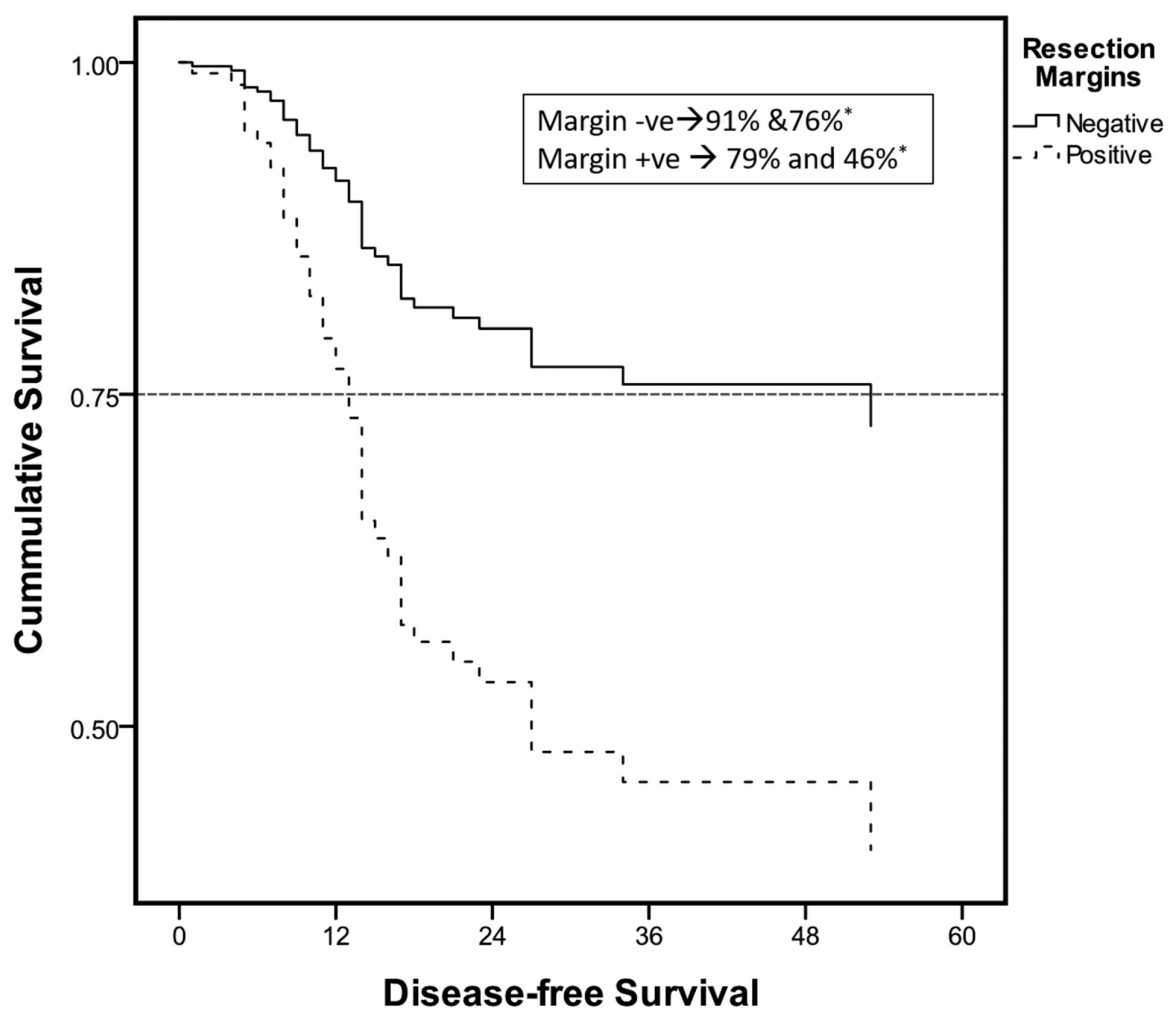

FIGURE 3: Cox regression survival curves: subgroup analysis

Survival curve: resection margin status.

*Disease-free survival at one and three years.

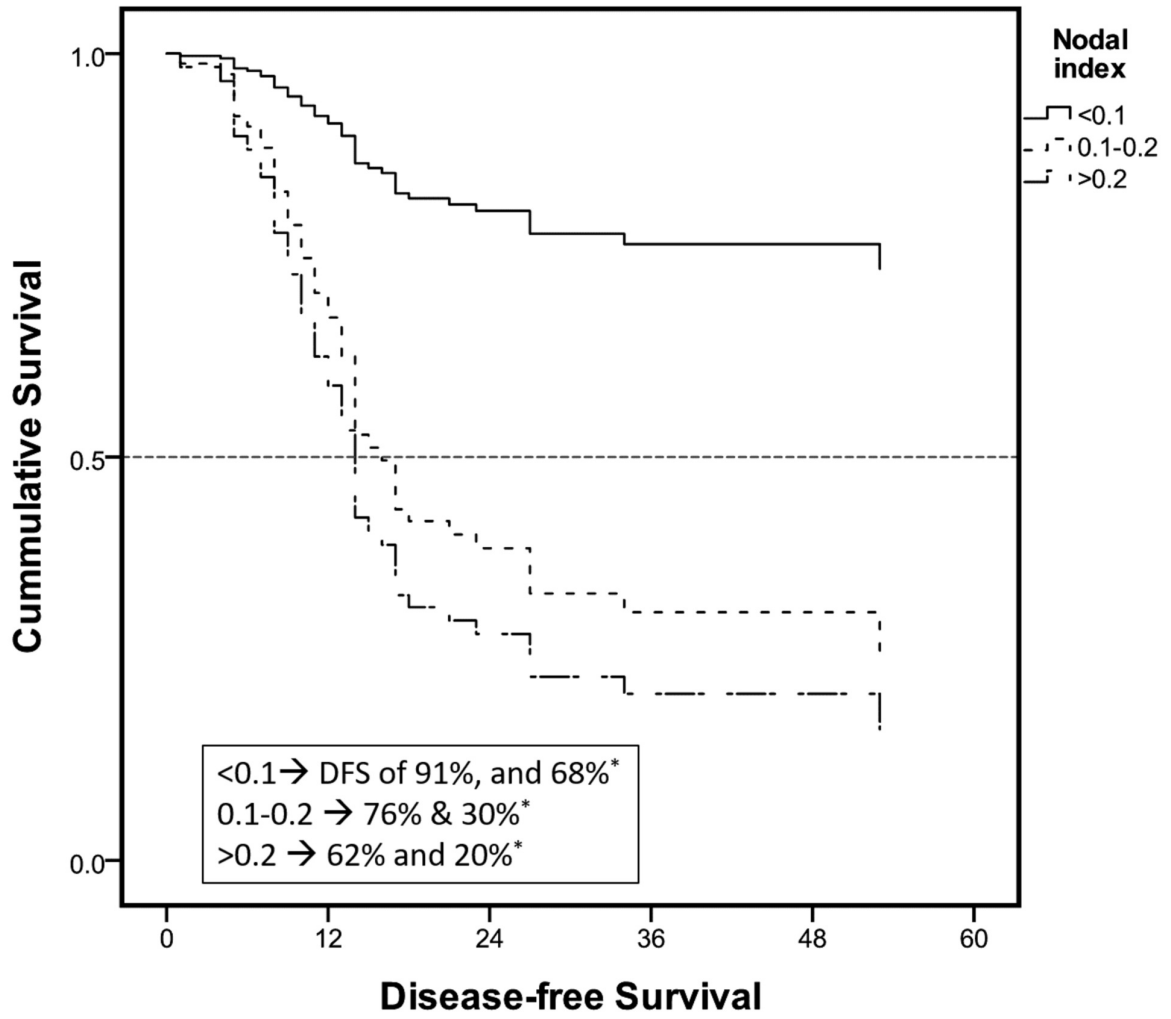




\section{Cureus}

FIGURE 4: Cox regression survival curves: subgroup analysis

Survival curve: nodal index shows the relative degree of impact on DSF of significant prognostic factors.

*DFS at one and three years

DFS, disease-free survival

\begin{tabular}{|c|c|c|c|}
\hline \multirow{3}{*}{ Survival Duration (in Months) } & \multicolumn{3}{|l|}{ Mean + SE } \\
\hline & \multicolumn{3}{|l|}{ Median (IQR) } \\
\hline & Total & Recurrence & No Recurrence \\
\hline \multirow{2}{*}{ Overall disease-free survival } & $34.9+1.75$ & $14+1.29$ & $45.5+1.81$ \\
\hline & $33(14-50.3)$ & $12.5(8-17)$ & $43.5(32-59.3)$ \\
\hline \multirow{2}{*}{ Time to recurrence } & \multicolumn{3}{|l|}{$13.3+1.25$} \\
\hline & \multicolumn{3}{|l|}{$12(8-16.3)$} \\
\hline
\end{tabular}

\section{TABLE 4: Cox survival statistics}

SE, standard error; IQR, interquartile range

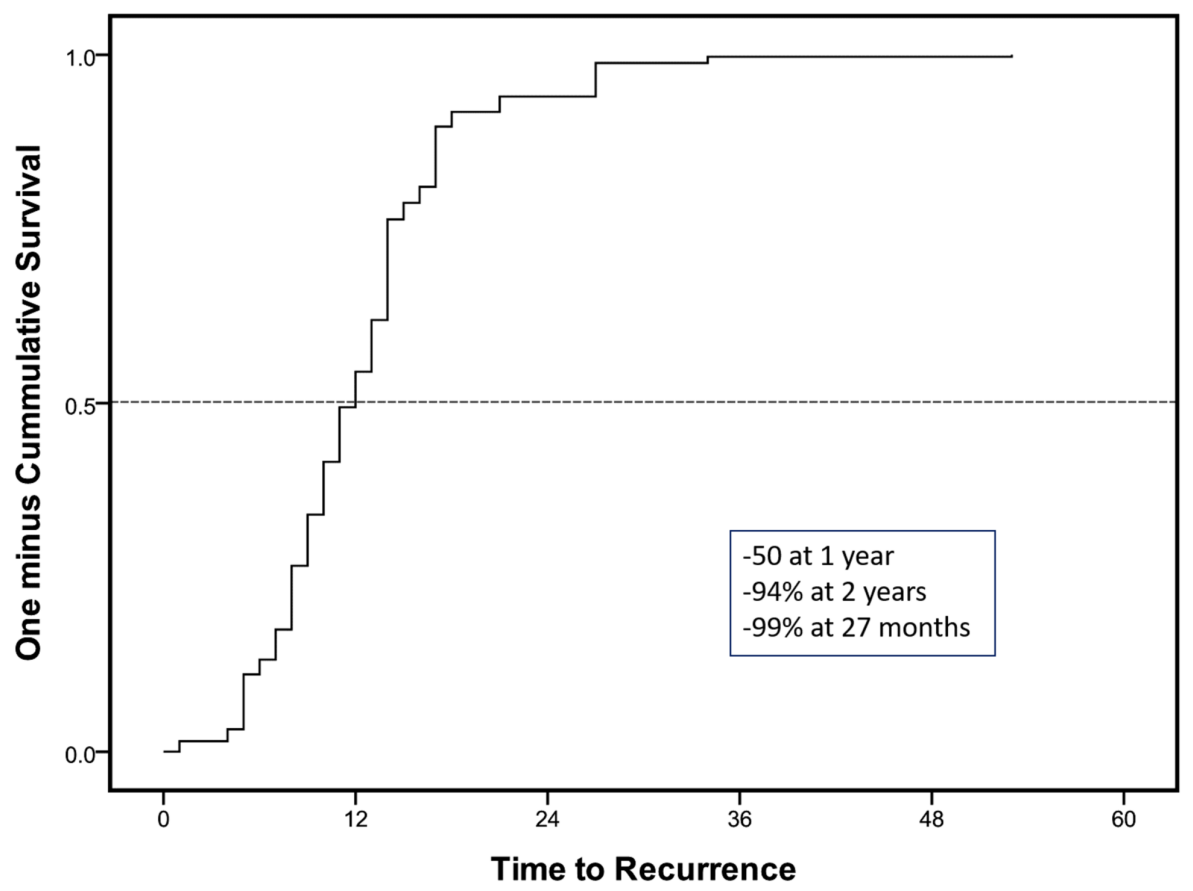

FIGURE 5: Cox regression survival functions for time to recurrence at the mean of covariates

Disease-Free Survival

Hazard ratio 


\section{Cureus}

Age $<55$ years vs.

$>55$ years

1.21

0.608

$0.59-2.45$

0.83

0.718

$0.30-2.28$

Gender: men vs.

Women

BMI: 18-25 vs.

$>25$

$<18$

1

0.993

$0.41-2.47$

0.68

0.569

$0.19-2.53$

0.408

$0.60-3.50$

0.732

$0.22-2.87$

Comorbidity index 0-1 vs.

$>1$

0.026

$1.16-10.52 \quad 1.01$

0.987

$0.21-4.86$

Histological subtype: SCCA vs.

Adenocarcinoma

1.35

0.627

$0.41-4.47$

3.8

0.136

$0.66-22.00$

Tumor grade well differentiated vs:

Moderately differentiated

1.19

0.796

$0.32-4.51$

0.38

0.317

$0.06-2.51$

Poorly differentiated

$0.18-3.54$

0.97

0.975

0.17-5.55

Tumor initial radiological stage: early vs.

Late (Illa and above)

2.05

0.098

$0.88-4.80$

1.84

0.326

$0.54-6.25$

Tumor location: Mid-esophageal vs.

Siewert type 1

0.2

Siewert type 2

0.47

0.006

$0.007-0.63$

0.94

$0.09-2.57$

0.24

0.942

$0.16-5.61$

Neoadjuvant type: Carboplatin-paclitaxel based XRt vs.

$\begin{array}{ll}\text { 5-FU based XRt } & 0.6 \\ \text { Magic protocol } & 0.53 \\ \text { Incomplete } & 0.7\end{array}$

Type of surgery conventional vs.

Minimally invasive

Hybrid

0.6

0.53

0.74

0.385

0.218

$0.03-2.31$

Waiting time for surgery: standard vs.

Delayed

1.28

0.47

$0.66-2.50$

0.57

0.307

0.19-1.69

Length of hospital stay $<11$ days vs.

$>11$ days

1.13

0.775

$0.48-2.68$

0.83

0.783

$0.22-3.13$

Post-operative: no vs.

Major complications

1.49

0.432

$0.55-3.99$

1.23

0.793

$0.27-5.61$

Complete pathological response vs.

Partial

2.78

0.006

$1.33-5.80$

2.45

0.099

0.84-7.09

Oncological complete resection vs.

Margin positive

0.004

$1.50-8.68$

1.99

0.21

$0.68-5.81$

Nodal index $<0.1 \mathrm{vs}$

$0.1-0.2$

4.35

$>0.2$

5.71
$1.72-11.04 \quad 1.9$

1.65-19.73 $\quad 4.04$
0.285

$0.59-6.16$

0.006

0.071

0.89-18.34 
TABLE 5: Cox regression analysis for disease-free survival and time to recurrence

$\mathrm{CI}$, confidence interval; BMI, body mass index; SCCA, squamous cell carcinoma; XRt, radiation therapy

\section{Discussion}

Our data suggest that in post-esophagectomy patients who are treated in combination with neoadjuvant treatment modalities, the only significant predictors for a higher risk to recurrence are higher nodal index, a positive resection margin, and residual disease. An additional risk factor for a poor DFS was a greater comorbidity index score.

A number of studies have analyzed factors influencing recurrence in patients with esophageal cancer postsurgical treatment with or without neoadjuvant chemoradiation therapy. In our series, the rate of recurrence was $33.1 \%$, which was comparable to the reported recurrence rate of other studies $(42.4 \%$ and $59.2 \%)[9,15]$. Lee et al. in their series of 465 resectable esophageal cancer patients identified the following variables as independent predictors of recurrence: performance status greater than 0 , poor tumor differentiation, en bloc resection, and advanced pathological stages (II/III/IV), on a multivariate regression model adjusted for $\mathrm{P}$ stage. Although they also found induction therapy to be an independent factor, unlike our patient population, more than $50 \%$ of their patients did not receive any neoadjuvant treatment [9]. In another similar study of 1,002 consecutive squamous cell carcinoma patients, Xu et al. concluded that the variables associated with a higher rate of recurrence by multivariate analyses were gender (hazard ratio [HR]: 1.7; 95\% confidence interval [CI]: 1.2 2.5; P = 0.002), depth of invasion (HR: 1.4; 95\% CI: 1.21 .6 ; P < 0.001), and lymph node involvement (HR: 1.4; 95\% CI: 1.3 1.5; P < 0.001) [15]. They excluded patients who received neoadjuvant treatment, and the patient population was considerably non-homogenous based on their adjuvant protocols. Our study failed to establish any association with the performance status and degree of tumor differentiation or gender with the rate and time of recurrence.

In our series, the median DFS was 33 months. While one- and three-year DFS was $90 \%$ and $72 \%$, respectively, it was a predicting factor that was statistically associated with a higher DFS and included CR, negative resection margins, a low nodal index, and a low comorbidity index. Lee et al. showed independent predictors of DFS to be the same as predictors of risk to recurrence in their series except for the histopathological tumor grade [9]. Eng et al. analyzed factors associated with overall survival after a trimodality treatment in esophageal adenocarcinoma. On Cox regression analysis, characteristics associated with a decreased overall survival included tumor stage, lymphovascular invasion, positive surgical margins, age, and comorbidity index. In addition, their results have demonstrated that post-operative adjuvant chemotherapy is also associated with increased survival in node-positive sub-group [12]. Another study by Yuequan et al. examined 1,553 cases of esophageal squamous cell carcinoma with a heterogeneous population of approximately $50 \%$ receiving adjuvant chemotherapy or XRt and the rest receiving upfront surgery. In this particular series, with an overall survival of $43.7 \%$ at three years, the independent prognostic factors they identified in addition to the stage of the cancer were the degree of tumor differentiation and family history of esophageal cancer [16].

The subject of optimal lymph node harvest has been a matter of constant debate. When the total number of resected nodes was examined as a categorical variable by Altorki et al. in their series of 264 patients without neoadjuvant therapy, for node-positive patients, the death hazard was significantly reduced for those with a higher total number of lymph nodes examined $(\mathrm{P}<0.05)$. While for node-negative patients, a significantly reduced hazard was present only when more than 40 nodes were resected $(H R=0.23 ; P=0.01)$ [17]. In this particular study, for multivariate and Cox regression analysis, the nodal index was used instead as a more relevant measure of recurrence [5,18]. Conversely, a more recent sub-group analysis of the Shapiro et al. failed to show any association of the total number of lymph nodes harvested with the survival in the patient population who received neoadjuvant chemoradiation therapy prior to surgery $[6,19]$.

Since the management protocol of esophageal cancer as far as standard tri-modality treatment is concerned stays independent of the tumor histological subtype, our study included both adenocarcinoma and squamous cell carcinoma patients. Moreover, for most of the cases, it is the disease stage and the patient's fitness level to undergo the protracted treatment course that define the treatment strategy. In our study, tumor histological subtype, as well as tumor location and degree of differentiation, failed to show any impact on any of the studied oncological parameters.

The rate of CR in this particular study from our center, keeping into consideration that it did not include patients who progressed on neoadjuvant treatment, was $55.2 \%$. The recurrence rate among the CR group was $20 \%$ as compared with the overall recurrence rate among patients with residual disease of $49.3 \%$. The percentage of CR among operated cases of squamous was $63 \%$, and the recurrence rate among this particular group was 26.7, making the rising question of wait-and-watch policy in this particular set of the population more conceivable [6]. CR among adenocarcinoma population was only $26.5 \%$. 
In our study, a waiting time of more than 6 weeks after neoadjuvant chemotherapy and more than 12 weeks after chemoradiation was considered as delayed, although a cutoff of eight weeks is usually used in the literature to study perioperative complications. In several studies, a long wait has been linked with a higher CR rate $[1-3,20]$. This was used to justify a delay in esophagectomy beyond eight weeks for patients who have not yet recovered from chemoradiation $[21,22]$. In our series, the median time to surgery after completion of neoadjuvant chemoradiation was 13 weeks (IQR = 11-18), and it remains an insignificant variable in terms of time to recurrence and DFS.

The identified risk factors of advanced pathological tumor $(\mathrm{T})$ and nodal $(\mathrm{N})$ stage, along with histopathological differentiation, have been proven significant determinants of recurrence-free survival for other solid organ malignancies [23-25]. This necessitates their further evaluation with more rigorous study protocols in order to assign this high-risk group either to a better surveillance protocol or to offer them additional treatment to decrease the likelihood of recurrence [12,26]. Until then, these results can motivate clinicians to remain critical in their patient selection and evaluate their clinical practice.

\section{Conclusions}

Our data suggest that it is the final histopathology report, elaborating upon the disease response to neoadjuvant treatment in terms of final pathological $\mathrm{T}$ and $\mathrm{N}$ stage, along with the surgical quality in terms of oncologically complete resection and good lymph node harvest, that determines the prognosis of nonmetastatic esophageal cancer patients after a completed tri-modality treatment. Our study concludes that histopathological stage, nodal status, and resection margin status are of utmost importance in determining the recurrence of resectable esophageal cancer in patients who had neoadjuvant chemoradiation therapy.

\section{Additional Information \\ Disclosures}

Human subjects: All authors have confirmed that this study did not involve human participants or tissue. Animal subjects: All authors have confirmed that this study did not involve animal subjects or tissue. Conflicts of interest: In compliance with the ICMJE uniform disclosure form, all authors declare the following: Payment/services info: All authors have declared that no financial support was received from any organization for the submitted work. Financial relationships: All authors have declared that they have no financial relationships at present or within the previous three years with any organizations that might have an interest in the submitted work. Other relationships: All authors have declared that there are no other relationships or activities that could appear to have influenced the submitted work.

\section{Acknowledgements}

We wish to thank Dr. Waleed Zafar and Dr. Farhana Badar for their contribution to the statistical design and review analysis.

\section{References}

1. Jemal A, Bray F, Center MM, Ferlay J, Ward E, Forman D: Global cancer statistics. CA Cancer J Clin. 2011, 61:69-90. 10.3322/caac.20107

2. National Comprehensive Cancer Network: Esophageal and esophagogastric junction cancer. (2019). Accessed: March 24, 2020: https://www.nccn.org/professionals/physician_gls/pdf/esophageal.pdf.

3. D’Journo XB, Thomas PA: Current management of esophageal cancer. J Thorac Dis. 2014, 6:253-64. 10.3978/j.issn.2072-1439.2014.04.16

4. Ychou M, Boige V, Pignon JP, et al.: Perioperative chemotherapy compared with surgery alone for resectable gastroesophageal adenocarcinoma: an FNCLCC and FFCD multicenter phase III trial. J Clin Oncol. 2011, 29:1715-21. 10.1200/TCO.2010.33.0597

5. Sjoquist KM, Burmeister BH, Smithers BM, Zalcberg JR, Simes RJ, Barbour A, Gebski V: Survival after neoadjuvant chemotherapy or chemoradiotherapy for resectable oesophageal carcinoma: an updated metaanalysis. Lancet Oncol. 2011, 12:681-92. 10.1016/S1470-2045(11)70142-5

6. Shapiro J, van Lanschot JJB, Hulshof MCCM, et al.: Neoadjuvant chemoradiotherapy plus surgery versus surgery alone for oesophageal or junctional cancer (CROSS): long-term results of a randomised controlled trial. Lancet Oncol. 2015, 16:1090-8. 10.1016/S1470-2045(15)00040-6

7. Swisher SG, Hofstetter W, Komaki R, et al.: Improved long-term outcome with chemoradiotherapy strategies in esophageal cancer. Ann Thorac Surg. 2010, 90:892-9. 10.1016/j.athoracsur.2010.04.061

8. Koshy M, Greenwald BD, Hausner P, et al.: Outcomes after trimodality therapy for esophageal cancer: the impact of histology on failure patterns. Am J Clin Oncol. 2011, 34:259-64. 10.1097/COC.0b013e3181e841ce

9. Lee PC, Mirza FM, Port JL, Stiles BM, Paul S, Christos P, Altorki NK: Predictors of recurrence and diseasefree survival in patients with completely resected esophageal carcinoma. J Thorac Cardiovasc Surg. 2011, 141:1196-206. 10.1016/j.jtcvs.2011.01.053

10. Annual cancer registry report-2016, of the Shaukat Khanum Memorial Cancer Hospital \& Research Center, Pakistan. (2016). Accessed: March 24, 2020: https://shaukatkhanum.org.pk/wpcontent/uploads/2015/06/acrr-2016.pdf.

11. Khan M, Muzaffar A, Syed AA, Khatak S, Khan AR, Ashraf MI: Changes in oncological outcomes: comparision of conventional and minimally invasive esophagectomy over ten years. Updates Surg. 2016, 68:343-9. 10.1007/s13304-016-0390-Z 
12. Eng OS, Nelson RA, Konstantinidis I, Chao J, Erhunmwunsee L, Raz DJ, Kim JY: Disparities in survival after trimodality therapy for esophageal adenocarcinoma. Dis Esophagus. 2018, 31:1. 10.1093/dote/doy020

13. Khan M, Urooj N, Syed AA, Khatak S, Ashraf MI, Batool S, Kazmi SA: Predicting factors for recurrence in esophageal cancer patients treated with neo-adjuvant therapy and surgery; a single institution analysis. I Cancer Allied Spec. 2017, 4:35. 10.37029/jcas.v3i4.159

14. Amin MB, Edge S, Greene F, et al.: AJCC Cancer Staging Manual. 8th Edition. Amin MB, Edge S, Greene F, et al. (ed): New York, Springer; 2017.

15. Xu Y, Chen Q, Yu X, Zhou X, Zheng X, Mao W: Factors influencing the risk of recurrence in patients with esophageal carcinoma treated with surgery: a single institution analysis consisting of 1002 cases. Oncol Lett. 2013, 5:185-90. 10.3892/ol.2012.1007

16. Yuequan J, Shifeng C, Bing Z: Prognostic factors and family history for survival of esophageal squamous cell carcinoma patients after surgery. Ann Thorac Surg. 2010, 90:908-13. 10.1016/j.athoracsur.2010.05.060

17. Altorki NK, Zhou XK, Stiles B, Port JL, Paul S, Lee PC, Mazumdar M: Total number of resected lymph nodes predicts survival in esophageal cancer. Ann Surg. 2008, 248:221-6. 10.1097/SLA.0b013e31817bbe59

18. Petrelli F, Borgonovo K, Barni S: The emerging issue of ratio of metastatic to resected lymph nodes in gastrointestinal cancers: an overview of literature. Eur J Surg Oncol. 2011, 37:836-47. 10.1016/j.ejso.2011.07.008

19. Koen Talsma A, Shapiro J, Looman CWN, et al.: Lymph node retrieval during esophagectomy with and without neoadjuvant chemoradiotherapy. Ann Surg. 2014, 260:786-93. 10.1097/SLA.0000000000000965

20. Franko J, Voynov G, Goldman CD: Esophagectomy timing after neoadjuvant therapy for distal esophageal adenocarcinoma. Ann Thorac Surg. 2016, 101:1123-30. 10.1016/j.athoracsur.2015.09.044

21. Kim JY, Correa AM, Vaporciyan AA, et al.: Does the timing of esophagectomy after chemoradiation affect outcome?. Ann Thorac Surg. 2012, 93:207-13. 10.1016/j.athoracsur.2011.05.021

22. Depypere L: The effect of time interval on esophagectomy after neoadjuvant treatment . Ann Transl Med. 2016, 4:117-117. 10.21037/atm.2016.01.28

23. Mirza AN, Mirza NQ, Vlastos G, Singletary SE: Prognostic factors in node-negative breast cancer: a review of studies with sample size more than 200 and follow-up more than 5 years. Ann Surg. 2002, 235:10-26. 10.1097/00000658-200201000-00003

24. Iizasa T, Suzuki M, Yoshida S, et al.: Prediction of prognosis and surgical indications for pulmonary metastasectomy from colorectal cancer. Ann Thorac Surg. 2006, 82:254-60. 10.1016/j.athoracsur.2006.02.027

25. Mehrkhani F, Nasiri S, Donboli K, Meysamie A, Hedayat A: Prognostic factors in survival of colorectal cancer patients after surgery. Color Dis. 2009, 11:157-61. 10.1111/j.1463-1318.2008.01556.x

26. Sudo K, Xiao L, Wadhwa R, et al.: Importance of surveillance and success of salvage strategies after definitive chemoradiation in patients with esophageal cancer. J Clin Oncol. 2014, 32:3400-5. 10.1200/JCO.2014.56.7156 